\title{
A proposito di Derrida: Biopolitica e immunità
}

Non sono né un conoscitore profondo dell'opera di Derrida né un suo allievo - qualsiasi significato si voglia dare a questa espressione. Lo dico con il rispetto che si deve ad un grande maestro del pensiero contemporaneo, ma anche con la consapevolezza di chi ha percorso, e percorre, una strada diversa dalla sua, pur apprezzandone tutta la forza e l'originalità. E' proprio da questa diversità, da questo scarto, che vorrei partire come chiave d'ingresso in un testo, come quello derridiano, non solo di straordinario interesse filosofico, ma anche di grande complessità interna. Per farlo userò uno dei suoi procedimenti preferiti e giustamente celebri: prenderò le mosse da quello che mi appare un sintomo, la traccia di un problema che la scrittura di Derrida non enuncia in quanto tale, ma allo stesso tempo nasconde e rivela. Mi riferisco al suo attacco a Giorgio Agamben contenuto nei seminari recentemente pubblicati con il titolo di La bestia e il sovrano. Non alludo al merito della polemica di Derrida - relativa alla dubbia sostenibilità dell'opposizione greca tra bios e zoe, così come è impostata da Agamben in una modalità effettivamente contestabile e contestata anche da altri - ma piuttosto alla sua forma, alla singolare virulenza impiegata in questa occasione da Derrida. La mia impressione è che il suo tono oltrepassi la normale divergenza di opinioni e lo stesso piano filosofico per sconfinare in quello personale, rivelando una insofferenza non limitata ad uno specifico argomento, ma che tocca e colpisce un intero stile di pensiero e di comportamento.

Secondo Derrida, nella sua volontà pervicace di attribuirsi un primato ermeneutico, una priorità storica, nell'elaborazione del paradigma biopolitico, Agamben avrebbe mancato di fare riferimento a chi, prima di lui e con argomenti anche più validi, aveva già tematizzato il rapporto differenziale tra vita animale e vita umana, e cioè Heidegger interprete di Aristotele. Da qui una polemica, nei confronti di Agamben, che per intere pagine alterna il tono della severità a quello dell'ironia. Quello che ci si può chiedere è cosa porti un autore, come appunto Derrida, solitamente generoso nei confronti dei suoi contemporanei, ad assumere un simile atteggiamento. Cosa determina questo singolare inasprimento di tono? Cosa spinge Derrida a superare i confini di una normale divergenza d'opinione? Chi, o cosa, egli vuole effettivamente colpire? E perché? 
La mia impressione è che Derrida abbia un altro, o un maggiore, obiettivo polemico rispetto a quello dichiaratovale a dire Foucault e quello che Foucault oggi rappresenta nel dibattito filosofico contemporaneo. Non solo, ma mi pare che ciò riveli una sua difficoltà a confrontarsi con lui su un terreno che per troppo tempo, se non da sempre, ha visto lo stesso Derrida più arretrato, o quantomeno non collocato in prima linea, vale a dire quello della vita biologica nei suoi rapporti con la politica. Quanto al primo punto si conosce - fin dalla famosa polemica degli anni Sessanta sul cogito cartesiano a proposito della Storia della follia - la distonia profonda, lessicale prima ancora che argomentativa, che separa Derrida da Foucault. Due autori spesso assimilati nella vulgata filosofica degli anni Settanta e Ottanta, ma in realtà fortemente distanti nelle intenzioni, nello stile, negli effetti di senso prodotti dalle loro opere. Ricordo ancora una sera a Venezia c'era appunto anche Agamben - in cui Jean-Luc Nancy, sappiamo quanto legato a Derrida, arrivò a sostenere che Foucault non è un filosofo, ma non più che uno storico, tra le nostre proteste, che invece gli attribuivamo, ed ancora gli attribuiamo un posto eminente nella filosofia novecentesca. Se il Novecento, come Foucault disse, può essere stato un secolo deleuziano, non è escluso che quello in corso sia, filosoficamente parlando, un secolo foucaultiano.

Senza adesso soffermarci su questa valutazione, evidentemente opinabile e controvertibile, richiamo l'episodio come una spia della distanza mai colmata, e quasi dell'allergia teoretica, che sia Derrida sia Nancy hanno in più occasioni manifestato nei confronti di ciò, che, proprio a partire a Foucault, ha assunto il nome di biopolitica. La verità è che entrambi - Derrida e Nancy - sensibili come sono ai passaggi di fase e anche alla dinamica del- la recezione internazionale, non potevano non avvertire il sensibile spostamento della french theory, cioè della diffusione americana del pensiero francese, dall'ambito dalla decostruzione a quello della biopolitica, evidente per chi si affacci nelle biblioteche dei maggiori campus delle università americane o legga gli ultimi fascicoli di "Diacritics" o di "Critical Inquiry".

Se, per tornare al merito delle questioni, si ricorda che già ne Le parole e le cose Foucault aveva individuato nella vita biologica uno dei tre trascendentali, cioè delle categorie costitutive, accanto al lavoro e al linguaggio, dell'episteme moderna, si può forse partire da qui per rintracciare un filo, e anche il punto originario, della presa di distanza di Derrida nei confronti del discorso foucaultiano e in genere del lessico biopolitico. Ancora preso - se posso esprimermi in maniera forse troppo sintetica - nella svolta linguistica che, già dagli anni Trenta del secolo scorso, concentrava sul linguaggio, o sul suo rovescio, la scrittura, lo sguardo della filosofia continentale ed analitica, Derrida non ha colto in tutta la sua rilevanza la 'svolta' successiva che, a partire dagli anni Sessanta, tendeva a sostituire all'orizzonte trascendentale del linguaggio quello, in realtà non più trascendentale in senso kantiano, ma, per così dire, storico-ontologico, della vita. Naturalmente sto radicalizzando un contrasto che andrebbe assai più sfumato e argomentato. Certo, Derrida ha lavorato a lungo sulla categoria di vita, in connessione con quella di morte - la-vita-la-morte - anche sulle tracce di Freud, oltre che di Heidegger, congiunti tra loro in Speculare su Freud. Ma egli si riferisce alla vita in un senso inassimilabile, o obliquo, rispetto a quello centrato, già a partire da Nietzsche, della categoria di biopolitica. Si potrebbe dire, anche qui abbreviando forse troppo un passaggio più complesso, che Derrida, come appunto Heidegger e 
Freud, pensi la vita dall'angolo di rifrazione della morte, anziché, come fa la biopolitica, pensare la morte dal punto di vista della vita.

E' vero che già col concetto di 'gramma' o di 'programma' Derrida allude, non solo in senso metaforico, al paradigma del vivente. Ma anche in questo caso senza tematizzare il passaggio decisivo che, ad un certo punto, comincia a fare della vita biologica insieme l'oggetto e il soggetto prevalente del potere. Naturalmente in un autore ricco e profondo come Derrida si può sempre trovare tutto ciò che si cerca - spunti, allusioni, illuminazioni che vanno in ogni direzione. La sua forza teoretica è tale da illuminare per contrasto anche ciò che lascia in ombra, anche il rovescio cavo di ciò che analizza frontalmente. Ma resta la sensazione - la mia sensazione - di uno scarto, e anche di un limite, che in qualche modo lo trattiene al di qua di una soglia epistemologica, gli preclude il transito in un diverso orizzonte di senso - Derrida, nonostante la sua straordinaria potenza innovativa, resta dentro l'orizzonte heideggeriano, e prima ancora kantiano, anche quando comincia a correggerlo in direzione di Levinas; non penetra in quello che Gilles Deleuze avrebbe definito 'piano di immanenza', resta sostanzialmente estraneo al discorso sulla centralità paradigmatica della vita. Si tratta di qualcosa come un'opacità - o un punto cieco - rispetto a quanto a Foucault, ma già a Nietzsche, appariva un'evidenza: la presenza sempre più estesa della vita biologica in tutti gli ambiti della vita contemporanea e la conseguenza che ciò determina anche nella pratica contemporanea della filosofia.

Una riprova di questo scarto analitico, di questo retrait teoretico di fronte a un passaggio d'epoca di tale rilievo, la vedo nel modo con egli ha successivamente impostato il rapporto tra comunità ed immunità. $\mathrm{O}$ meglio non lo ha impostato, separandone le sematiche, non valorizzando adeguatamente l'elemento chiave che le lega e le mette in tensione reciproca, vale a dire il munus. Certo, anche in questo caso sappiamo tutti che Derrida ha a lungo e lucidamente lavorato sul concetto d dono e di dono avvelenato, ma senza connettere il munus al cum della relazione, senza pensare il comune e l'immune e soprattutto la dialettica che insieme li congiunge e li disgiunge. Ciò - questa opzione per così dire riduttiva - lo conduce ad una doppia conseguenza. Da un lato a rifiutare il tema, o almeno il lessico, della comunità. Dall'altro a non cogliere tutte le implicazioni e gli effetti di senso della questione dell'immunità, una volta separata da quella della comunità e non inscritta a pieno titolo dentro l'orizzonte della vita biologica.

Partiamo dal primo punto. Come è noto, soprattutto in Politiche dell 'amicizia, Derrida rifiuta l'uso del termineconcetto di 'comunità'. E non solo di quello fatto proprio dai vari comunitari otto e novecenteschi - da Toennies ai neocomunitarsti americani, ancora presi nel lessico organicistico della Gemeinschaft o portatori di istanze regressive e localistiche. La riserva di Derrida si rivolge anche a coloro, da Bataille a Nancy, passando per Blanchot, che in anni più recenti hanno decostruito la mitologia organicistica e appropriativa del comunitarismo americano a favore di una differente concezione della comunità. Benché legato a questi pensatori da tanti fili, Derrida se ne distanzia in maniera netta proprio sulla questione decisiva della comunità. Perché? Come mai? 'Comunità', a suo dire, è parola, termine, concetto, troppo compromesso dalla sua storia recente e meno recente per poter assumere un significato diverso ed opposto a quello che a lungo gli si è dato e che in qualche modo gli si continua a 
dare. Le stesse formule che tentano di distanziarsene o di decostruirlo dialetticamente - come quelle di 'comunità inconfessabile' o 'inoperosa' o di 'coloro che non hanno comunità' - ricadono, per Derrida, inevitabilmente preda del significato che intendono negare. Anzi tale negazione, o autonegazione, gli appare il sintomo più evidente della incapacità di uscire da qualcosa, da un circolo semantico, che ha i caratteri di un rimosso della tradizione occidentale - la potenza dell'indiviso, più forte di tutte le differenze, le diversioni, le divergenze e perciò stesso destinata a riprodurre la genealogia del sangue fraterno.

Che dire di ciò? Come valutare questa presa di distanza, lessicale e concettuale, dal linguaggio della comunità che spinge Derrida lontano da qualcosa che per molti di noi è stato, assai più di un tema, un modo nuovo di fare filosofia? Intanto si potrebbe obiettare che se non fosse possibile lavorare ad una trasformazione semantica di parole che hanno una storia complessa e ambivalente, non dovremmo più usare neanche un termine come quello di 'democrazia' sul quale Derrida è invece, giustamente, sempre tornato. Ma proprio perciò l'unica via che mi pare capace di ridare senso a una parola irrinunciabile come, nonostante tutto, resta quella di 'comunità' è un'interrogazione capace di penetrare a fondo nella sua scatola nera, vale a dire nella fonte di senso custodita nella sua origine etimologica. Solamente in questo modo - tornando ad un'analisi radicale del termine munus - da cui quello di communitas deriva, è possibile spezzare la crosta che si è formata lungo la sua storia, liberare la parola $\mathrm{e}$ il concetto di comunità da quel significato appropriativo che l'ha stravolto e pervertito nel suo opposto. Se è vero che, per quanto indicibile, inconfessabile, inoperosa, la comunità non riesce a liberarsi del tutto del suo significato moderno, che rischia sempre di essere riafferrata da ciò da cui pure prende le distanze, ciò non vale per la communitas, che non si limita a rovesciare a posteriori un significato canonico, ma fin dall'inizio ne origina uno diverso ed ampiamente compatibile con ciò che lo stesso Derrida intende per amicizia - un niente-in-comune che unisce nella distanza, un vuoto o una faglia costituita dal non-poter-essere tale dell'individuo o dell'indiviso.

Direi, anzi, qualcosa di più. Mentre il concetto di amicizia, di philia, per quanto decostruito, resta inevitabilmente esposto ad un tonalità umanistica, ciceroniana, di carattere etico-morale, la communitas, intesa nel senso appena definito, ha una connotazione meno recuperabile dal linguaggio del soggetto e dalla sua logica della presupposizione perché originariamente attinente al linguaggio della esposizione. In fondo l'amicizia, comunque la si declini, resta inevitabilmente situata all'interno di un lessico intersoggettivo. Ad essere amici sono pur sempre soggetti individualmente definiti e rivolti l'uno al riconoscimento dell'altro. Mentre i membri della communitas - in quanto costituiti dal munus comune - non sono riconducibili a una figura della soggettività, né all'ego né all'alter, ma ad un taglio originario che apre ed altera il soggetto in tutta la sua estensione.

Un riscontro anche esterno di quanto dico - del deficit teoretico derivato dalla mancata elaborazione del concetto di 'comunità' inteso nel suo senso originario - lo trovo nella teorizzazione avanzata nelle ultime opere di Derrida dell'idea di 'immunità' o meglio di 'autoimmunità', che avrebbe dovuto, o potuto, accostarlo alla riflessione biopolitica e che invece lo tiene lontano da essa, per come è da lui elaborato. Ma andiamo con ordine. L'autoimmunità - cui Derrida si riferisce per la prima volta nel suo saggio sulla religione e poi, più diffusamente, in Stati canaglie e 
nella intervista con la Borradori sull'11 settembre - allude alla potenza dissolutiva di un apparato difensivo tanto forte da rivolgersi contro se stesso distruggendosi, come accade appunto nelle cosiddette malattie autoimmuni. La globalizzazione, intesa come una sorta di contaminazione generalizzata, ha scatenato una reazione di rigetto, una sindrome autoimmunitaria, destinata a devastare lo stesso sistema che vorrebbe proteggere. Come questa analisi colga un tratto decisivo dell'esperienza contemporanea è sotto gli occhi di tutti. Non a caso negli stessi anni, anche se in modo indipendente gli uni dagli altri, in Italia, in Germania e negli Stati Uniti i filosofi più attenti a quella che Foucault chiamava 'ontologia dell'attualità' - da Sloterdijk a Donna Haraway, ad altri - hanno preso a lavorare su questo concetto, anche se a partire da presupposti differenti. Personalmente penso che la categoria, il dispositivo, di immunità, per esprimere tutte le sue potenzialità, vada inquadrata nel doppio riferimento alla semantica della comunità e a quella della biopolitica. Derrrida, pur cogliendo la rilevanza del tema e fornendone una significativa interpretazione, non fa né l'una né l'altra cosa. Non connette il paradigma immunitario né alla questione della communitas né a quello della biopolitica. E' per questo, credo, che da lui l'immunità è sempre e soltanto intesa nel senso, distruttivo ed autodistruttivo, dell'autoimmunità. Ciò deriva dal fatto che non è legata né ad una elaborazione concettuale del munus comune né a quella del nesso, potenzialmente affermativo, tra politica e bios. Fuori da questo doppio riferimento l'immunità finisce per essere interpretata sempre in chiave negativa ed escludente - condannata in anticipo a battere contro se stessa.

Ora, come invece appare dagli studi più recenti, anche di immunologia medica, il sistema immunitario è qualcosa di più articolato e plurivoco: protezione $e$ negazione della vita. Ciò vuol dire che, insieme e in contraddizione con la sua figura negativa, esso implica anche una potenza positiva - altrimenti non sarebbero possibili né i trapianti d'organo né le gravidanze, entrambi fenomeni non solo consentiti, ma in un certo senso resi possibili soltanto dai sistemi immunitari del nostro corpo. Se reimmesso entro l'orizzonte della vita biologica, oltre, e forse più, che barriera difensiva e offensiva contro l'altro da sé, il sistema immunitario può essere inteso anche come un filtro, come una cassa di risonanza, in cui il sé si relaziona con l'altro, il medesimo con il diverso, l'interno con l'esterno. Non so se l'assenza di questo lato - affermativo - della problematica immunitaria in Derrida sia riconducibile a quella diffidenza, o quantomeno non integrale assunzione dell'orizzonte della vita biologica, cui ho accennato all'inizio. Al fatto che nella sua opera sterminata e geniale Derrida non conferisca un posto adeguato al fenomeno del vivente, come invece fa l'altra linea della filosofia francese contemporanea che va da Bergson a Canguilhem, da Foucault a Deleuze.

Anche nel già citato libro sull'amicizia il tema del bios, della vita, viene pensato da Derrida sempre in negativo - in rapporto alla categoria, potenzialmente violenta, di fraternità. Ma se le sue considerazioni sono assolutamente pertinenti quanto alla deriva etnocentrica della fratellanza, ciò non vuol dire che la vita, il bios, in quanto tale, non contenga anche una potenza affermativa, costitutiva, come emerge dalla grande tradizione spinoziano-nietscheana e poi dagli studi più recenti sulla biopolitica. Naturalmente questi elementi affermativi nella politica della vita non vanno enfatizzati, come non lo sono mai stati dallo stesso Foucault. La politica della vita è sempre esposta ad una relazione complessa ed 
anche ambigua con una logica di morte, al rischio di rovesciarsi in tanatopolitica. Ciò non toglie, tuttavia, che è difficile lavorare su categorie, come quelle di immunità $o$ comunità fuori da un quadro teoretico aperto a una considerazione anche affermativa del paradigma di vita. Si potrebbe arrivare a dire che il discrimine fondamentale della filosofia novecentesca passi tra coloro che pensano la vita a partire dall'orizzonte della morte - come Heidegger, Freud e lo stesso Derrida - e coloro, come Nietzsche, Bergson e Foucault, che pensano la morte a partire dall'orizzonte della vita. Che non presuppongono la morte alla vita e anzi la fanno finita con la logica stessa del Presupposto. Con l'idea che ogni origine abbia alle spalle un Inizio indicibile e irrappresentabile che la tormenta risucchiandola in una morte già da sempre presente. Forse il senso stesso della filosofia contemporanea sta nel rompere quest'incantesimo, nel ricacciare nelle tenebre questo antichissimo fantasma, nel liberarsi insieme dal Precedente e dall'Adveniente, a favore di un presente senza resti, di una coincidenza assoluta della vita con se stessa. Naturalmente queste mie osservazioni nei confronti di un grande filosofo quale è stato Derrida non vanno intese come una critica, e, tantomeno, come una presa di distanza nei confronti di un maestro indimenticabile, ma come la traccia di una differenza che, mentre separa, al contempo unisce percorsi diversi nella forma della condivisione di un orizzonte comune. Nulla del lavoro svolto da tutti noi in questi anni sarebbe stato lo stesso senza il riferimento ad un testo - quello di Derrida - che non solo penetra profondamente nell'orizzonte del nostro tempo, ma che ancora a lungo segnerà le vie del pensiero. 